\title{
Tree Diversity and Their Regeneration in the Sacred Groves of Virajpet, Central Western Ghats, India
}

\author{
Devi A.G. PRASAD, Shwetha BHARATHI*
}

University of Mysore,DOS in Environmental Science, Manasagangotri, Mysore-570006, Karnataka,
India; agdprasad@yahoo.com; shwethabharathib@gmail.com ("correspondingauthor)

\begin{abstract}
Sacred groves are one of the finest examples of informal way of conserving the forest wealth. Baseline data collection of their diversity, distribution and regeneration capacity becomes necessary for the management and conservation of these undisturbed forest patches. In this context, the present investigation was carried out using random quadrat method in the sacred groves of Virajpet, Karnataka, India. A total of 132 tree species belonging to 113 genera and 45 families were identified within five sacred groves. Higher basal area $(51.73-85.65 \mathrm{~m} / \mathrm{ha})$ and tree density (453.33-515.9 individuals/ha) were observed as compared to other forests of Western Ghats region. The present investigation has revealed a healthy regeneration of tree species. Seedling and sapling composition differed to some extent from the mature tree species composition which could be used in predicting the future possibilities. Protection and conservation of such sacred groves should be of interest, for better regeneration of the rich diversity they harbour.
\end{abstract}

Keywords: conservation, density, Kodagu, regenerates, tropical forests

\section{Introduction}

The diversity of tree species is a fundamental component of total biodiversity in many ecosystems as they are the ecosystem engineers that provide resources and habitats for almost all other forest organisms. In tropical forests, trees form the major structural and functional basis of forest ecosystems and can serve as robust indicators of changes and stressors of the landscape (Jayakumar and Nair, 2013). Understanding their diversity, distribution pattern and regeneration would help in evaluating the complexity and potential resources of the forest. Further, management of a forest requires baseline data on understanding its composition, the effects of past impacts on the present status and the relationship of the forest with surrounding land uses (Devi and Yadav, 2006).

Sacred groves, as an example of informal way of protecting and managing the forest ecosystems, have been proved to sustain rich biodiversity as well as greater ecological services (Raghavendra and Kushalappa, 2011). In fact, they represent patches of virgin forest left as it is, in the name of local deity and are protected by the local people due to their cultural and religious beliefs and taboos that the deities reside in them (Khan et al., 2008). As they are protected for centuries, sacred groves harbour climax vegetation and relic specimens of plant world. They are known to possess endemic as well as threatened species. Ecological significance of sacred groves can be explained by the biological diversity found within them. The conservation of biodiversity is of foremost importance. Also, conservational attitude towards natural resources passed on through cultural beliefs to the younger generations is of immense importance.

Sacred groves of Karnataka are mainly found on the Western Ghats region. Though studies on assessment of biodiversity and management system of these sacred groves are taken up, vegetation analysis and regeneration studies are limited. Therefore, the present investigation was undertaken to evaluate the diversity, distribution and regeneration status of arboreal species of five sacred groves of Virajpet, Central Western Ghats.

\section{Materials and Methods}

Studyarea

Kodagu is one of the highly wooded Western Ghats regions of Karnataka, India. The altitude of the district ranges between 850 and $1,745 \mathrm{~m}$. The average temperature varies from $14.2^{\circ} \mathrm{C}$ (winter) to $28.6{ }^{\circ} \mathrm{C}$ (summer). The average annual rainfall is $2,725.5 \mathrm{~mm}$, received mainly from South-Western monsoon concentrated during the months of June to September. Soil type of the area varies greatly due to geological heterogeneities, although sandy loamy soil type was found in the study sites. It is majorly composed of semi-evergreen to evergreen forests (Keshavamurthy and Yoganarasimhan, 1990).

The study was undertaken in five sacred groves of Virajpet, Kodagu District (Fig. 1). Higher density of sacred groves is found in this district, making it a 'hotspot of sacred groves' in the world (Raghavendra and Kushalappa, 2011). The district has 1,214 sacred groves covering an area of 2,550 ha, of which 508 sacred groves covering an area of 872.13 ha are in Virajpet. Of the five sacred groves studied (Table 1), SG1 has concrete temple 
constructed, SG2 and SG3 have medium sized temples, whereas remaining groves still enjoy nature worship. The deities in the groves are worshipped once a year during March/April.

\section{Data collection and analysis}

Field exploration was undertaken to record the tree species composition of the sacred groves. Quadrats of $20 \times 20 \mathrm{~m}$ dimension were laid randomly to enumerate the mature trees covering $5 \%$ of total area. Stems having GBH (girth at breast level $-1.34 \mathrm{~m}$ height) $\geq 30 \mathrm{~cm}$ were counted as adults/trees. Identification of species was made using authentic floras (Saldanha, 1984; Pascal and Ramesh, 1987; Keshavamurthy and Yoganarasimhan, 1990; Saldanha, 1996; Poornika et al., 2011) and voucher specimens have been deposited in Biodiversity Conservation Laboratory, DOS in Environmental Science, University of Mysore, Mysore, India.

Regeneration studies were carried out by laying down plots of $2 \times 2 \mathrm{~m}$ size randomly. In each plot, all tree species $\leq 30 \mathrm{~cm} \mathrm{GBH}$ were considered as regenerates and enumerated separately into seedlings ( $<40 \mathrm{~cm}$ height) and saplings $(>40 \mathrm{~cm}$ height and $<30$ $\mathrm{cm} \mathrm{GBH})$. Regeneration status of each tree species was evaluated considering the number of seedlings and saplings based on Shankar (2001), with some modifications: good regeneration - if seedlings > saplings > adults; fair regeneration - if seedlings > or < saplings > or < adults; poor regeneration - if the species survive only at sapling stage or only as seedling stage (seedlings and saplings may be less or more or equal to adults); no regeneration if a species was present only in adult form; reappearing/immigrating- if the species has no adults, but only seedlings or saplings.

Floristic diversity was measured by using Simpson Index of Diversity (1-D) (Simpson, 1949), Shannon and Wiener's Index of Diversity (H) (Shannon and Wiener, 1963) and Pielou's Evenness Index (J') (Pielou, 1966). Importance Value Index (IVI) of each tree species was determined (Curtis and McIntosh, 1950) calculating frequency, density and basal area. Similarity

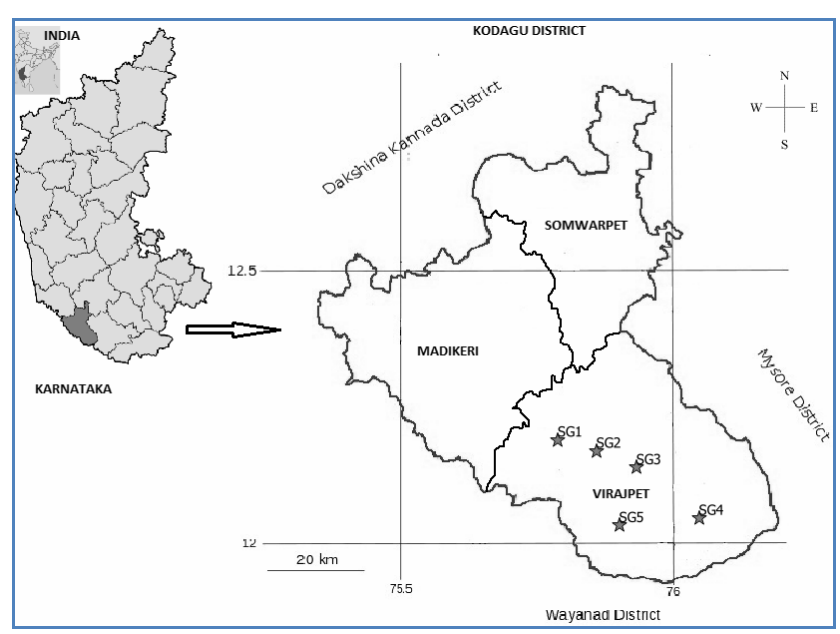

Fig. 1. Map of study area was determined by Sorenson's Similarity Index (Sorenson, 1948). Degree of disturbance was assessed in terms of 14 parameters such as cut stumps, grazing, fire, weeds, roads, footpath, domestic animal dung, wild animal dung, collection of firewood, canopy opening, encroachment, conversion, sanskritization and impact of pilgrim's activities. Four levels of disturbance were given to each parameter namely absent (0), low (1), medium (2) and high (3). For each sacred grove, the degree of disturbance was expressed in-terms of all fourteen parameters as percentage of Combined Disturbance Index (CDI) (Gunaga et al., 2013). CDI values were compared with basal area, number of species, tree density, seedling density and sapling density of each sacred grove for possible correlation.

\section{Results}

A total of 132 tree species belonging to 113 genera and 45 families were identified in the five sacred groves of Virajpet, Kodagu district, India. Of these, 105 species had mature individuals and 27 species were recorded only in regenerating stage (reappearing/immigrating status). Of the 45 families, Lauraceae was represented by the maximum of eleven species, followed by Euphorbiaceae, Fabaceae (ten species each) and Moraceae (nine species). Twenty one families were represented by single species (Table 2).

Higher species richness was observed in all the sacred groves. However, lowest and highest values were recorded in SG5 and SG2 respectively. Overall, Shannon's index value was 3.3228, Simpson's index value was 0.9424 and Pielou's index value was 0.8586 . On average, basal area of $68.5721 \mathrm{~m}^{2} / \mathrm{ha}$ and tree density of 477.48 individuals/ha was recorded (Table 2 ). All the five sacred groves showed a reverse-J shaped curve for different girth classes (Fig. 1). Overall, $61.84 \%$ of individuals found in the study sites were within $30-90 \mathrm{~cm}$ girth class. Only $5.14 \%$ of mature individuals were in the group with $>270 \mathrm{~cm}$ girth class.

Reverse J-shaped curve was observed in SG1, SG3 and SG4 for four regeneration classes (Fig. 3). Percentage of class I was lesser than class II and class III in SG2 and SG4 respectively altering the reverse J-shaped curve. Regeneration status of each tree species was tabulated (Table 2). Overall, $42.07 \%$ of tree species showed regeneration (Fig. 4), of which $9.31 \%$ of species had good regeneration, $13.20 \%$ of species had fair regeneration and $19.55 \%$ of species had poor regeneration status, while $26.08 \%$ of tree species had no regeneration and $31.82 \%$ of species had no mature individuals. Seedling and sapling densities among the sacred groves varied greatly. Higher seedling and sapling densities $\left(855.35\right.$ regenerates $/ 100 \mathrm{~m}^{2}$ and 641.96 regenerates $/ 100 \mathrm{~m}^{2}$ respectively) were recorded from SG5 and lowest from SG4.

Among the groves, Sorenson's similarity index value ranged from $57.55 \%$ (SG1 and SG4) to 69.86\% (SG3 and SG4) (Table 4). It was observed that nine species namely Antidesma montanum, Artocarpus beterophyllus,

Table 1. Geographical details of the study area

\begin{tabular}{|c|c|c|c|c|}
\hline Name of the studied sacred grove & Residing deity & Area (acres) & Location & Elevation \\
\hline Betoli sacred grove (SG1) & Bhadrakali & 23.83 & 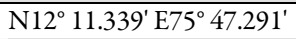 & $882 \mathrm{~m}$ \\
\hline Kolthodu sacred grove (SG2) & Ayyappa & 29.2 & $\mathrm{~N} 12^{\circ} 10.107^{\prime} \mathrm{E} 75^{\circ} 51.550^{\prime}$ & $842 \mathrm{~m}$ \\
\hline Huduru sacred grove (SG3) & Ayyappa & 16.54 & $\mathrm{~N} 12^{\circ} 08.288^{\prime} \mathrm{E} 75^{\circ} 55.965^{\prime}$ & $877 \mathrm{~m}$ \\
\hline Thaila sacred grove (SG4) & Ayyappa & 25.1 & $\mathrm{~N} 12^{\circ} 02.707^{\prime} \mathrm{E} 76^{\circ} 02.862^{\prime}$ & $877 \mathrm{~m}$ \\
\hline Birunani sacred grove (SG5) & Ayyappa & 15.62 & $\mathrm{~N} 12^{\circ} 01.908^{\prime} \mathrm{E} 75^{\circ} 54.069^{\prime}$ & $824 \mathrm{~m}$ \\
\hline
\end{tabular}


362

Artocarpus birsutus, Celtis tetrandra, Caryota urens, Cinnamomum malabatrum, Dimocarpus longan, Glochidion ellipticum and Mangifera indica were common to all the sacred groves with good density of mature individuals. However, of these, Dimocarpus longan showed good regeneration with higher number of seedlings and saplings in all the sacred groves.
Betoli sacred grove (SGI)

A total of 67 tree species belonging to 56 genera and 33 families were recorded from SG1 sampling area, among which 16 species were represented by one individual (singleton species) and 17 species had no mature individuals. Tree density and basal area were recorded to be 515.9 individuals/ha and $64.14 \mathrm{~m}^{2} /$ ha respectively.

Table 2. Importance Value Index values and regeneration status of tree species among the five sacred groves in Virajpet, Kodagu district

\begin{tabular}{|c|c|c|c|c|c|c|c|c|c|c|c|c|}
\hline \multirow{2}{*}{ Slno } & \multirow{2}{*}{ Botanical name } & \multirow{2}{*}{ Family name } & \multicolumn{2}{|c|}{ SG1 } & \multicolumn{2}{|c|}{ SG2 } & \multicolumn{2}{|c|}{ SG3 } & \multicolumn{2}{|c|}{ SG4 } & \multicolumn{2}{|c|}{ SG5 } \\
\hline & & & IVI & RS & IVI & RS & IVI & $\mathrm{RS}$ & IVI & $\mathrm{RS}$ & IVI & RS \\
\hline 1 & $\begin{array}{l}\text { Holigarna arnottiana Hook. } \\
\text { f. }\end{array}$ & Anacardiaceae & 1.2025 & $\mathrm{~N}$ & - & - & - & - & - & - & - & - \\
\hline 2 & $\begin{array}{l}\text { Holigarna grahamii (Wight) } \\
\text { Kurz. }\end{array}$ & Anacardiaceae & 2.4831 & $\mathrm{P}$ & 16.9601 & $\mathrm{P}$ & - & $\mathrm{R} / \mathrm{I}$ & 13.8901 & $\mathrm{P}$ & 6.0373 & $\mathrm{P}$ \\
\hline 3 & Holigarna nigra Bourd. & Anacardiaceae & 1.1589 & $\mathrm{~N}$ & 2.2718 & $\mathrm{~F}$ & - & $\mathrm{R} / \mathrm{I}$ & - & $\mathrm{R} / \mathrm{I}$ & - & - \\
\hline 4 & Mangiffraindica $\mathrm{L}$. & Anacardiaceae & 2.7168 & $\mathrm{~F}$ & 3.5552 & G & 7.029 & $\mathrm{~F}$ & 1.1586 & $\mathrm{P}$ & 3.4546 & $\mathrm{~F}$ \\
\hline 5 & $\begin{array}{l}\text { Nothopegia racemoosa } \\
\text { (Dalz.) Ramam. }\end{array}$ & Anacardiaceae & 18.6243 & G & 6.9872 & G & - & $\mathrm{R} / \mathrm{I}$ & 7.2105 & G & 22.1141 & G \\
\hline 6 & Spondiaspinnata (L.f.) Kurz & Anacardiaceae & - & - & 4.1579 & $\mathrm{~N}$ & 3.4119 & $\mathrm{~N}$ & 8.8182 & $\mathrm{P}$ & 1.9819 & $\mathrm{~N}$ \\
\hline 7 & $\begin{array}{l}\text { Goniothalamus cardioupetalus } \\
\text { (Dalz.)Hook. f.\& Thoms. }\end{array}$ & Annonaceae & - & - & - & $\mathrm{R} / \mathrm{I}$ & - & $\mathrm{R} / \mathrm{I}$ & - & $\mathrm{R} / \mathrm{I}$ & - & $\mathrm{R} / \mathrm{I}$ \\
\hline 8 & Alstoniascholaris (L.)R Br. & Apocynaceae & 1.5129 & $\mathrm{~N}$ & 2.4454 & $\mathrm{~N}$ & - & - & - & - & - & - \\
\hline 9 & $\begin{array}{l}\text { Tabernaemontana heyneana } \\
\text { Wall. }\end{array}$ & Apocynaceae & 1.4023 & $\mathrm{~N}$ & 1.1855 & $\mathrm{~N}$ & - & $\mathrm{R} / \mathrm{I}$ & 1.9485 & $\mathrm{~N}$ & 1.9422 & $P$ \\
\hline 10 & Caryotaurens $\mathrm{L}$. & Arecaceae & 19.2563 & $\mathrm{~F}$ & 2.1588 & $\mathrm{P}$ & 18.4811 & $\mathrm{~F}$ & 0.9946 & $\mathrm{P}$ & 21.7639 & $P$ \\
\hline 11 & Vernonia arborea Ham. & Asteraceae & - & - & - & $\mathrm{R} / \mathrm{I}$ & - & - & - & - & - & - \\
\hline 12 & $\begin{array}{l}\text { Stereospermum colais (Buch.- } \\
\text { Ham. Ex Dillw.) }\end{array}$ & Bignoniaceae & - & - & - & - & 21.9126 & $\mathrm{P}$ & 5.7744 & $\mathrm{~N}$ & 9.8832 & $\mathrm{~N}$ \\
\hline 13 & $\begin{array}{l}\text { Pajanelia longifolia (Willd.) } \\
\text { K.Schum. }\end{array}$ & Bignoniaceae & 1.2585 & $\mathrm{~N}$ & - & - & - & - & - & - & - & - \\
\hline 14 & Canarium strictum Roxb. & Burseraceae & 29.0486 & $P$ & 19.8749 & $P$ & - & $\mathrm{R} / \mathrm{I}$ & 5.358 & $\mathrm{P}$ & 2.6222 & $\mathrm{~N}$ \\
\hline 15 & $\begin{array}{l}\text { Euonymusindicus Heyne ex } \\
\text { Wall. }\end{array}$ & Celastraceae & 6.7661 & $\mathrm{~F}$ & 1.109 & $\mathrm{~F}$ & - & $\mathrm{R} / \mathrm{I}$ & - & $\mathrm{R} / \mathrm{I}$ & - & $\mathrm{R} / \mathrm{I}$ \\
\hline 16 & Euonymussps & Celastraceae & - & $\mathrm{R} / \mathrm{I}$ & - & - & - & - & - & - & - & - \\
\hline 17 & $\begin{array}{l}\text { Cassine glauca (Rottb.) } \\
\text { Kuntze }\end{array}$ & Celastraceae & - & - & - & $\mathrm{R} / \mathrm{I}$ & - & - & - & $\mathrm{R} / \mathrm{I}$ & - & $\mathrm{R} / \mathrm{I}$ \\
\hline 18 & $\begin{array}{l}\text { Calophyllum polyanthum } \\
\text { Wall. ex Choisy. }\end{array}$ & Clusiaceae & - & - & - & - & - & - & 5.905 & $\mathrm{P}$ & - & - \\
\hline 19 & $\begin{array}{l}\text { Garciniagummi-gutta }(\mathrm{L} \text {.) } \\
\text { Robson. }\end{array}$ & Clusiaceae & - & $\mathrm{R} / \mathrm{I}$ & - & $\mathrm{R} / \mathrm{I}$ & - & $\mathrm{R} / \mathrm{I}$ & - & - & - & $\mathrm{R} / \mathrm{I}$ \\
\hline 20 & Mesuaferrea $\mathrm{L}$. & Clusiaceae & - & - & 3.7262 & $\mathrm{~N}$ & - & - & - & - & - & - \\
\hline 21 & $\begin{array}{l}\text { Garcinia indica (Thouars) } \\
\text { Choisy }\end{array}$ & Clusiaceae & - & - & 0.9754 & $\mathrm{~N}$ & - & - & - & - & - & - \\
\hline 22 & Terminaliapaniculata Roth & Combretaceae & - & - & - & - & 2.05 & $\mathrm{~N}$ & - & - & - & - \\
\hline 23 & $\begin{array}{l}\text { Mastixia arborea (Wight) } \\
\text { Bedd. }\end{array}$ & Cornaceae & - & - & 4.0671 & $\mathrm{~F}$ & - & - & 3.8641 & G & - & - \\
\hline 24 & $\begin{array}{l}\text { Dichapetalum gelonioides } \\
\text { (Roxb.) Engl. }\end{array}$ & Dichapetalaceae & - & $\mathrm{R} / \mathrm{I}$ & - & - & - & - & - & - & - & $\mathrm{R} / \mathrm{I}$ \\
\hline 25 & $\begin{array}{l}\text { Hopeaponga (Dennst.) } \\
\text { Mabberly }\end{array}$ & Dipterocarpaceae & - & - & - & - & 1.4688 & $\mathrm{P}$ & - & - & - & - \\
\hline 26 & Vateria indica $\mathrm{L}$ & Dipterocarpaceae & - & - & - & - & - & - & - & - & 28.1918 & G \\
\hline 27 & Diospyros montana Roxb. & Ebenaceae & 1.2748 & $\mathrm{~N}$ & - & - & - & - & - & $\mathrm{R} / \mathrm{I}$ & - & - \\
\hline 28 & Diospyros candolleana Wight & Ebenaceae & - & - & 2.0929 & $\mathrm{~N}$ & - & - & 1.2474 & $\mathrm{~N}$ & - & - \\
\hline 29 & Diospyroscrumenata Thw. & Ebenaceae & - & - & - & $\mathrm{R} / \mathrm{I}$ & - & - & 0.9491 & $\mathrm{~N}$ & - & - \\
\hline 30 & Elaeocarpusserratus $\mathrm{L}$. & Elaeocarpaceae & 1.2705 & $\mathrm{~N}$ & 1.2238 & $\mathrm{~N}$ & 3.4668 & $\mathrm{~N}$ & - & - & - & - \\
\hline 31 & $\begin{array}{l}\text { Elaeocarpus tuberculatus } \\
\text { Roxb. }\end{array}$ & Elaeocarpaceae & - & - & - & - & 3.5183 & $\mathrm{~N}$ & - & - & 3.5329 & $\mathrm{~F}$ \\
\hline 32 & $\begin{array}{l}\text { Antidesmamontanum } \\
\text { Blume. }\end{array}$ & Euphorbaceae & 5.1093 & $\mathrm{~N}$ & 6.6833 & G & 1.6138 & $\mathrm{P}$ & 4.9892 & $\mathrm{P}$ & 6.0639 & $\mathrm{P}$ \\
\hline 33 & Bischofiajavanica Bl. & Euphorbaceae & - & - & - & - & - & $\mathrm{R} / \mathrm{I}$ & 1.3022 & $\mathrm{P}$ & - & - \\
\hline 34 & Glochidion ellipticum Wight. & Euphorbaceae & 6.0024 & $\mathrm{P}$ & 5.5751 & $\mathrm{P}$ & 3.6079 & $\mathrm{~N}$ & 5.1798 & $\mathrm{~N}$ & 1.9925 & $\mathrm{~N}$ \\
\hline 35 & $\begin{array}{l}\text { Mallotusphilippensis(Lam) } \\
\text { Muell-Arg. }\end{array}$ & Euphorbaceae & - & - & 0.9582 & $\mathrm{P}$ & 1.4688 & $\mathrm{~F}$ & 7.978 & $\mathrm{~F}$ & 7.6063 & $\mathrm{P}$ \\
\hline 36 & $\begin{array}{l}\text { Mallotustetracoccus (Roxb.) } \\
\text { Kurz. }\end{array}$ & Euphorbaceae & 2.5175 & $\mathrm{~N}$ & 6.2706 & $\mathrm{P}$ & 36.7543 & $\mathrm{~N}$ & 5.4663 & $\mathrm{~N}$ & - & - \\
\hline 37 & Cleidionjavanicum $\mathrm{Bl}$. & Euphorbaceae & - & $\mathrm{R} / \mathrm{I}$ & - & $\mathrm{R} / \mathrm{I}$ & - & - & 20.614 & G & - & - \\
\hline 38 & $\begin{array}{l}\text { Macarangapeltata (Roxb.) } \\
\text { Mueller }\end{array}$ & Euphorbaceae & - & - & - & - & - & - & 2.3768 & $\mathrm{~N}$ & 8.9266 & $\mathrm{~N}$ \\
\hline 39 & $\begin{array}{l}\text { Aporusalindleyana }(\mathrm{Wt} \text { ) } \\
\text { Bail. }\end{array}$ & Euphorbaceae & - & - & - & $\mathrm{R} / \mathrm{I}$ & - & $\mathrm{R} / \mathrm{I}$ & - & $\mathrm{R} / \mathrm{I}$ & - & $\mathrm{R} / \mathrm{I}$ \\
\hline 40 & $\begin{array}{l}\text { Antidesmamenasu Miq. Ex } \\
\text { Tul. }\end{array}$ & Euphorbaceae & - & - & 7.7997 & $\mathrm{~N}$ & - & - & - & - & - & - \\
\hline 41 & U1 & Euphorbaceae & - & - & 1.0902 & $\mathrm{~N}$ & - & - & - & - & - & - \\
\hline 42 & $\begin{array}{l}\text { Acrocarpus fraxinifolius } \\
\text { Wight \& Arn. }\end{array}$ & Fabaceae & - & $\mathrm{R} / \mathrm{I}$ & - & $\mathrm{R} / \mathrm{I}$ & - & - & - & - & - & $\mathrm{R} / \mathrm{I}$ \\
\hline 43 & $\begin{array}{l}\text { Archidendron monadelphum } \\
\text { (Roxb.) Nielson. }\end{array}$ & Fabaceae & - & - & - & - & - & - & - & - & 9.1067 & $\mathrm{P}$ \\
\hline
\end{tabular}




\begin{tabular}{|c|c|c|c|c|c|c|c|c|c|c|c|c|}
\hline 45 & Pongamia pinnata $(\mathrm{L}$.$) Pierre$ & Fabaceae & - & - & - & - & 7.8627 & $\mathrm{P}$ & - & - & - & - \\
\hline 46 & Albiziachinensis(Osb.) Merr. & Fabaceae & - & - & - & - & 5.3207 & $\mathrm{~N}$ & - & - & - & - \\
\hline 47 & $\begin{array}{l}\text { Buteamonosperma (Lam.) } \\
\text { Taub. }\end{array}$ & Fabaceae & - & - & - & - & 1.5716 & $\mathrm{~N}$ & - & - & - & - \\
\hline 48 & Erythrina variegata $\mathrm{L}$. & Fabaceae & - & - & 1.0939 & $\mathrm{~N}$ & - & - & - & - & - & - \\
\hline 49 & Humboldtiabrunonis Wall. & Fabaceae & - & $\mathrm{R} / \mathrm{I}$ & - & - & - & - & - & - & - & - \\
\hline 50 & $\begin{array}{l}\text { Saracaasoca (Roxb.) de } \\
\text { Wilde. }\end{array}$ & Fabaceae & - & - & - & - & - & - & 5.2178 & $\mathrm{~F}$ & - & - \\
\hline 51 & Baubinia purpurea $\mathrm{L}$. & Fabaceae & - & - & - & $\mathrm{R} / \mathrm{I}$ & - & - & - & - & - & - \\
\hline 52 & Flacourtia Montana Graham & Flacourtiaceae & - & - & - & - & - & $\mathrm{R} / \mathrm{I}$ & 1.9337 & $\mathrm{P}$ & - & - \\
\hline 53 & $\begin{array}{l}\text { Scolopiacrenata (Wight \& } \\
\text { Arn.)Clos }\end{array}$ & Flacourtiaceae & 2.6953 & $\mathrm{P}$ & 4.3869 & $\mathrm{P}$ & - & $\mathrm{R} / \mathrm{I}$ & 5.5362 & $\mathrm{P}$ & 1.935 & $\mathrm{~N}$ \\
\hline 54 & $\begin{array}{l}\text { Hydnocarpuspentandra } \\
\text { (Buch-Ham) Oken }\end{array}$ & Flacourtiaceae & 4.1827 & $P$ & 10.0081 & $\mathrm{P}$ & 23.1801 & $\mathrm{~F}$ & 25.5181 & $\mathrm{~F}$ & - & $\mathrm{R} / \mathrm{I}$ \\
\hline 55 & $\begin{array}{l}\text { Apodytes dimidiata E. Meyer } \\
\text { ex Arn. }\end{array}$ & Icacinaceae & 1.1752 & $\mathrm{~N}$ & 0.9537 & $\mathrm{~N}$ & 1.471 & $\mathrm{~N}$ & 1.0524 & $\mathrm{~N}$ & - & - \\
\hline 56 & $\begin{array}{l}\text { Nothapodytesnimmoniana } \\
\text { (Graham)Mabberley }\end{array}$ & Icacinaceae & - & - & - & $\mathrm{R} / \mathrm{I}$ & - & $\mathrm{R} / \mathrm{I}$ & - & $\mathrm{R} / \mathrm{I}$ & 1.9751 & $\mathrm{P}$ \\
\hline 57 & $\begin{array}{l}\text { Actinodaphnemalabarica } \\
\text { Balak. }\end{array}$ & Lauraceae & - & - & 3.1586 & $\mathrm{~F}$ & - & $\mathrm{R} / \mathrm{I}$ & - & $\mathrm{R} / \mathrm{I}$ & 2.8365 & $\mathrm{~F}$ \\
\hline 58 & $\begin{array}{l}\text { Cinnamomum malabatrum } \\
\text { (Burm. F.) Bl. }\end{array}$ & Lauraceae & 22.2897 & $\mathrm{~F}$ & 9.2068 & $\mathrm{~F}$ & 3.9461 & G & 9.4092 & G & 9.5722 & G \\
\hline 59 & $\begin{array}{l}\text { Litseafloribunda (Bl.) } \\
\text { Gamble }\end{array}$ & Lauraceae & 2.8485 & $\mathrm{~F}$ & 1.9292 & $\mathrm{~N}$ & - & $\mathrm{R} / \mathrm{I}$ & 5.5774 & $\mathrm{P}$ & 6.0134 & $\mathrm{P}$ \\
\hline 60 & $\begin{array}{l}\text { Litsea oleoides (Meissn.) } \\
\text { Hook.f. }\end{array}$ & Lauraceae & - & $\mathrm{R} / \mathrm{I}$ & 5.7392 & $\mathrm{~N}$ & - & - & 2.2189 & $\mathrm{~N}$ & 2.2474 & $\mathrm{P}$ \\
\hline 61 & $\begin{array}{l}\text { Neolitseazeylanica (Nees) } \\
\text { Merr., Philip. }\end{array}$ & Lauraceae & 1.7071 & $\mathrm{~N}$ & - & - & - & - & 2.3178 & $\mathrm{~N}$ & - & - \\
\hline 62 & $\begin{array}{l}\text { Persea macrantha (Nees) } \\
\text { Kosterm. }\end{array}$ & Lauraceae & 2.3222 & $\mathrm{~N}$ & - & - & 1.9964 & $\mathrm{~N}$ & - & - & - & $\mathrm{R} / \mathrm{I}$ \\
\hline 63 & $\begin{array}{l}\text { Cryptocarya bourdillonii } \\
\text { Gamble. }\end{array}$ & Lauraceae & 4.7525 & G & - & - & - & - & - & - & - & $\mathrm{R} / \mathrm{I}$ \\
\hline 64 & Cryptocaryasps & Lauraceae & - & - & - & - & - & - & - & - & - & $\mathrm{R} / \mathrm{I}$ \\
\hline 65 & $\begin{array}{l}\text { Litsea laevigata (Nees) } \\
\text { Gamble }\end{array}$ & Lauraceae & - & $\mathrm{R} / \mathrm{I}$ & - & - & - & - & - & - & - & - \\
\hline 66 & Litsea mysorensis Gamble & Lauraceae & 1.1458 & $\mathrm{P}$ & 0.9524 & $\mathrm{~F}$ & - & - & - & - & - & $\mathrm{R} / \mathrm{I}$ \\
\hline 67 & $\begin{array}{l}\text { Cinnamomumverum } \\
\text { J.S.Presl }\end{array}$ & Lauraceae & - & - & - & $\mathrm{R} / \mathrm{I}$ & - & - & 3.0842 & $\mathrm{~N}$ & - & - \\
\hline 68 & Leeaindica (Burm. F.) Merr. & Leeaceae & 1.329 & $\mathrm{~F}$ & - & $\mathrm{R} / \mathrm{I}$ & - & $\mathrm{R} / \mathrm{I}$ & - & $\mathrm{R} / \mathrm{I}$ & - & $\mathrm{R} / \mathrm{I}$ \\
\hline 69 & Fagraea ceilanica Thumb. & Loganiaceae & 1.1656 & $\mathrm{P}$ & - & - & - & - & - & - & - & - \\
\hline 70 & $\begin{array}{l}\text { Lagestroemiamicrocarpa } \\
\text { Wight }\end{array}$ & Lythraceae & - & - & - & - & 4.0111 & $\mathrm{~N}$ & 11.667 & $\mathrm{~N}$ & - & - \\
\hline 71 & Magnoliachampaka L. & Magnoliaceae & - & $\mathrm{R} / \mathrm{I}$ & 0.9511 & $\mathrm{~N}$ & 6.8693 & $\mathrm{P}$ & 1.9448 & $\mathrm{~N}$ & - & - \\
\hline 72 & $\begin{array}{l}\text { Memecylon malabaricum } \\
\text { (C.B.Clarke) Cogn. }\end{array}$ & Melastomataceae & 3.5277 & G & - & - & - & $\mathrm{R} / \mathrm{I}$ & - & - & - & $\mathrm{R} / \mathrm{I}$ \\
\hline 73 & $\begin{array}{l}\text { Memecylon umbellatum } \\
\text { Burm.f. }\end{array}$ & Melastomataceae & - & - & - & $\mathrm{R} / \mathrm{I}$ & - & - & - & - & - & - \\
\hline 74 & Memecylon edule Roxb. & Melastomataceae & - & - & - & $\mathrm{R} / \mathrm{I}$ & - & - & - & - & - & - \\
\hline 75 & Memecylon sps & Melastomataceae & - & - & - & $\mathrm{R} / \mathrm{I}$ & - & - & - & - & - & - \\
\hline 76 & $\begin{array}{l}\text { Aphanamixispolystachya } \\
\text { (Wall.) Parker. }\end{array}$ & Meliaceae & - & - & - & $\mathrm{R} / \mathrm{I}$ & 1.4732 & $\mathrm{~N}$ & - & - & - & - \\
\hline 77 & $\begin{array}{l}\text { Dysoxylum malabaricum } \\
\text { Bedd. ex Heirn }\end{array}$ & Meliaceae & 25.4298 & $\mathrm{~N}$ & - & - & - & - & - & - & - & - \\
\hline 78 & Melia composita Willd. & Meliaceae & - & - & - & 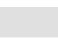 & 1.4732 & $\mathrm{P}$ & - & - & - & - \\
\hline 79 & $\begin{array}{l}\text { Trichilia connaroides (Wight } \\
\text { \& Arn.) Bentevelzen }\end{array}$ & Meliaceae & - & - & - & - & 1.4829 & G & 0.9491 & $\mathrm{~N}$ & - & - \\
\hline 80 & $\begin{array}{l}\text { Reinwardtiodendron } \\
\text { anamalaiense (Bedd.) Mabb. }\end{array}$ & Meliaceae & - & - & - & - & - & - & 6.5966 & G & - & - \\
\hline 81 & Aglaiabarberi Gamble & Meliaceae & - & - & 3.1639 & $\mathrm{~N}$ & - & - & - & - & - & - \\
\hline 82 & Antiaris toxicaria Lesch. & Moraceae & - & $\mathrm{R} / \mathrm{I}$ & 8.3969 & $\mathrm{~N}$ & - & - & - & - & - & - \\
\hline 83 & Artocarpusheterophyllus Lam. & Moraceae & 7.6116 & $\mathrm{~F}$ & 11.3435 & $\mathrm{~F}$ & 1.471 & $\mathrm{~F}$ & 2.2046 & $P$ & 20.9634 & $\mathrm{~F}$ \\
\hline 84 & Artocarpushirsutus Lam. & Moraceae & 2.3632 & $\mathrm{~F}$ & 9.2062 & $\mathrm{~F}$ & 4.9829 & G & 12.0628 & $\mathrm{~F}$ & 4.6637 & $\mathrm{~F}$ \\
\hline 85 & Ficus amplissima Smith & Moraceae & - & - & 1.214 & $\mathrm{~N}$ & 1.6085 & $\mathrm{~N}$ & 1.0109 & $\mathrm{~N}$ & 2.571 & $\mathrm{~N}$ \\
\hline 86 & FicushispidaL.f. & Moraceae & 1.2057 & $\mathrm{~F}$ & 2.8287 & $\mathrm{P}$ & 4.4157 & $\mathrm{~F}$ & 2.3187 & $\mathrm{~F}$ & - & - \\
\hline 87 & $\begin{array}{l}\text { Artocarpus gomeziamus Wall. } \\
\text { Ex Trec. }\end{array}$ & Moraceae & - & - & - & - & - & - & - & - & 2.0254 & $\mathrm{P}$ \\
\hline 88 & $\begin{array}{l}\text { Ficustijabela Rheede ex } \\
\text { Burm.F. }\end{array}$ & Moraceae & - & - & 8.3063 & $\mathrm{~N}$ & - & - & - & - & - & - \\
\hline 89 & Ficusexasperata Vahl. & Moraceae & - & - & & $\mathrm{R} / \mathrm{I}$ & - & - & - & - & - & - \\
\hline 90 & U1 & Moraceae & - & - & 0.9772 & $\mathrm{~N}$ & - & - & - & - & - & - \\
\hline 91 & Myristica dactaloides Gaertn. & Myristicaceae & 8.9227 & $\mathrm{P}$ & 6.1844 & $\mathrm{P}$ & - & - & - & $\mathrm{R} / \mathrm{I}$ & - & - \\
\hline 92 & Ardisia solonaceae Roxb. & Myrsenaceae & - & - & - & - & - & $\mathrm{R} / \mathrm{I}$ & - & $\mathrm{R} / \mathrm{I}$ & - & - \\
\hline 93 & Syzygium sps & Myrtaceae & 4.014 & $\mathrm{P}$ & - & $\mathrm{R} / \mathrm{I}$ & 1.4776 & $\mathrm{~F}$ & - & - & 2.0124 & $\mathrm{~N}$ \\
\hline 94 & $\begin{array}{l}\text { Syzygium hemisphericum } \\
\text { (Wight) Alston }\end{array}$ & Myrtaceae & - & $\mathrm{R} / \mathrm{I}$ & - & - & - & - & - & - & - & - \\
\hline 95 & $\begin{array}{l}\text { Syzygium zeylanicum }(\mathrm{L} .) \\
\text { DC. }\end{array}$ & Myrtaceae & - & $\mathrm{R} / \mathrm{I}$ & - & - & - & $\mathrm{R} / \mathrm{I}$ & 1.0606 & $\mathrm{~F}$ & - & - \\
\hline 96 & Syzygium cuminii (L)Skeels & Myrtaceae & - & & 28.1666 & $\mathrm{~F}$ & 14.7571 & $\mathrm{P}$ & 25.2512 & $\mathrm{~N}$ & 4.344 & $\mathrm{~N}$ \\
\hline 97 & $\begin{array}{l}\text { Chionanthusmala-lengi } \\
\text { (Dennst.) Green. }\end{array}$ & Oleaceae & - & - & 3.7364 & $\mathrm{~F}$ & 3.6984 & $\mathrm{~F}$ & 1.0133 & $\mathrm{P}$ & 1.9785 & $\mathrm{P}$ \\
\hline 98 & Olea dioica Roxb. & Oleaceae & 8.8503 & $\mathrm{P}$ & 0.9968 & $\mathrm{P}$ & 1.4688 & $\mathrm{P}$ & - & $\mathrm{R} / \mathrm{I}$ & 7.6894 & $\mathrm{~F}$ \\
\hline
\end{tabular}




\begin{tabular}{|c|c|c|c|c|c|c|c|c|c|c|c|c|}
\hline 99 & Oleapaniculata R. Br. & Oleaceae & - & - & - & - & - & - & 1.0047 & $\mathrm{~N}$ & - & - \\
\hline 100 & $\begin{array}{l}\text { Ziziphusxylopyrus (Retz.) } \\
\text { Willd }\end{array}$ & Rhamnaceae & - & - & - & - & - & - & - & $\mathrm{R} / \mathrm{I}$ & - & - \\
\hline 101 & $\begin{array}{l}\text { Carallia brachiata (Lour.) } \\
\text { Merr. }\end{array}$ & Rhizophoraceae & - & - & - & - & - & - & - & - & - & $\mathrm{R} / \mathrm{I}$ \\
\hline 102 & $\begin{array}{l}\text { Canthium dicoccum } \\
\text { (Gaertn.) Teijsm. \& Binn. }\end{array}$ & Rubiaceae & 1.1458 & $\mathrm{P}$ & - & - & - & - & - & - & - & - \\
\hline 103 & Ixora brachiata Roxb. & Rubiaceae & - & $\mathrm{R} / \mathrm{I}$ & - & $\mathrm{R} / \mathrm{I}$ & - & $\mathrm{R} / \mathrm{I}$ & - & $\mathrm{R} / \mathrm{I}$ & - & $\mathrm{R} / \mathrm{I}$ \\
\hline 104 & Pavettaindica $\mathrm{L}$. & Rubiaceae & - & - & - & - & - & $\mathrm{R} / \mathrm{I}$ & - & - & - & $\mathrm{R} / \mathrm{I}$ \\
\hline 105 & $\begin{array}{l}\text { Psychotria nigra (Gaert.) } \\
\text { Alston }\end{array}$ & Rubiaceae & - & $\mathrm{R} / \mathrm{I}$ & - & $\mathrm{R} / \mathrm{I}$ & - & $\mathrm{R} / \mathrm{I}$ & - & $\mathrm{R} / \mathrm{I}$ & - & - \\
\hline 106 & $\begin{array}{l}\text { Wendlandia thyrsoidea } \\
\text { (Roth.)Steud }\end{array}$ & Rubiaceae & - & - & - & - & - & - & 1.1732 & $\mathrm{~N}$ & - & - \\
\hline 107 & $\begin{array}{l}\text { Lxoranigricans R.Br.Ex } \\
\text { Wight \& Arn. }\end{array}$ & Rubiaceae & - & $\mathrm{R} / \mathrm{I}$ & - & $\mathrm{R} / \mathrm{I}$ & - & $\mathrm{R} / \mathrm{I}$ & - & $\mathrm{R} / \mathrm{I}$ & - & $\mathrm{R} / \mathrm{I}$ \\
\hline 108 & Meyna laxiflora Robyns & Rubiaceae & - & - & 1.9935 & $\mathrm{~N}$ & - & $\mathrm{R} / \mathrm{I}$ & 4.0909 & $P$ & 2.3828 & $\mathrm{P}$ \\
\hline 109 & Psychotria truncata Wall. & Rubiaceae & - & $\mathrm{R} / \mathrm{I}$ & - & - & - & $\mathrm{R} / \mathrm{I}$ & - & - & - & - \\
\hline 110 & Atalantiasps & Rutaceae & - & - & - & - & - & - & - & $\mathrm{R} / \mathrm{I}$ & - & - \\
\hline 111 & Euodialunu-ankenda Merr. & Rutaceae & 4.5147 & $\mathrm{P}$ & 5.9624 & $\mathrm{~N}$ & 1.551 & $\mathrm{~N}$ & 0.9985 & $\mathrm{~N}$ & 3.0441 & $\mathrm{~N}$ \\
\hline 112 & $\begin{array}{l}\text { Atalantiamonophylla }(\mathrm{L} .) \\
\text { DC. }\end{array}$ & Rutaceae & - & $\mathrm{R} / \mathrm{I}$ & - & $\mathrm{R} / \mathrm{I}$ & - & $\mathrm{R} / \mathrm{I}$ & - & - & - & $\mathrm{R} / \mathrm{I}$ \\
\hline 113 & $\begin{array}{l}\text { Veprisbilocularis (Wight \& } \\
\text { Arn.) Engl. }\end{array}$ & Rutaceae & - & - & 1.7146 & $\mathrm{~N}$ & - & - & 2.3204 & $\mathrm{~N}$ & - & - \\
\hline 114 & $\begin{array}{l}\text { Acronychia pedunculata (L.) } \\
\text { Miq. }\end{array}$ & Rutaceae & 5.6573 & $\mathrm{P}$ & - & $\mathrm{R} / \mathrm{I}$ & - & $\mathrm{R} / \mathrm{I}$ & 5.9701 & $\mathrm{~N}$ & 12.4148 & $\mathrm{~N}$ \\
\hline 115 & $\begin{array}{l}\text { Scleropyrum pentandrum } \\
\text { (Dennst.) Mabb }\end{array}$ & Santalaceae & 1.5129 & $\mathrm{~N}$ & - & - & - & $\mathrm{R} / \mathrm{I}$ & - & - & - & - \\
\hline 116 & Dimocarpuslongan Lour. & Sapindaceae & 28.2142 & G & 13.095 & G & 16.5608 & G & 5.6827 & G & 51.1393 & G \\
\hline 117 & $\begin{array}{l}\text { Herpullia arborea (Blanco) } \\
\text { Radlk. }\end{array}$ & Sapindaceae & 1.2153 & $\mathrm{~N}$ & - & - & - & - & 5.3519 & F & - & - \\
\hline 118 & $\begin{array}{l}\text { Sapindustrifoliatus auct. } \\
\text { Non.L }\end{array}$ & Sapindaceae & - & - & - & - & - & $\mathrm{R} / \mathrm{I}$ & 4.3247 & $\mathrm{~F}$ & - & - \\
\hline 119 & $\begin{array}{l}\text { Chrysophyllum roxburghii } \mathrm{G} \text {. } \\
\text { Don. }\end{array}$ & Sapotaceae & 1.1571 & $\mathrm{~F}$ & 12.1321 & $\mathrm{P}$ & 4.805 & $\mathrm{P}$ & 2.2491 & $\mathrm{~F}$ & - & $\mathrm{R} / \mathrm{I}$ \\
\hline 120 & Mimusupselengi L. & Sapotaceae & 2.7441 & $\mathrm{~N}$ & 1.8239 & $\mathrm{~N}$ & - & - & - & - & - & - \\
\hline 121 & Sterculia guttata Roxb. & Sterculiaceae & - & - & 2.3051 & $\mathrm{~F}$ & - & - & 7.288 & $\mathrm{~F}$ & - & $\mathrm{R} / \mathrm{I}$ \\
\hline 122 & $\begin{array}{l}\text { Symplocos macrophylla Wall. } \\
\text { ex A.DC }\end{array}$ & Symplocaceae & 2.4101 & $\mathrm{~F}$ & 1.986 & $\mathrm{P}$ & - & - & - & - & 4.7937 & G \\
\hline 123 & Eurya nitida Korth. & Theaceae & - & - & - & - & - & - & - & - & 6.3382 & $\mathrm{~N}$ \\
\hline 124 & Grewia tiliifolia Vahl. & Tiliaceae & - & - & - & - & 18.1611 & $\mathrm{P}$ & - & - & - & - \\
\hline 125 & $\begin{array}{l}\text { Aphananthe cuspidate (Bl) } \\
\text { Planch. }\end{array}$ & Ulmaceae & 7.325 & $\mathrm{~N}$ & 1.6127 & $\mathrm{~N}$ & 16.5963 & $\mathrm{~N}$ & 6.886 & $\mathrm{~F}$ & - & - \\
\hline 126 & Celtistetrandra Roxb. & Ulmaceae & 6.2938 & $\mathrm{P}$ & 1.9183 & $\mathrm{~F}$ & 2.9563 & $\mathrm{P}$ & 18.7388 & $\mathrm{~F}$ & 3.1142 & $\mathrm{P}$ \\
\hline 127 & Trema orientalis $(\mathrm{L}.) \mathrm{Bl}$. & Ulmaceae & 2.695 & $\mathrm{~N}$ & 1.5389 & $\mathrm{~N}$ & 10.0086 & $\mathrm{P}$ & - & - & - & - \\
\hline 128 & $\begin{array}{l}\text { Callicarpa tomentosa }(\mathrm{L} \text {.) } \\
\text { Murr. }\end{array}$ & Verbenaceae & 5.5043 & $\mathrm{~F}$ & 3.9582 & $\mathrm{P}$ & 1.808 & G & - & $\mathrm{R} / \mathrm{I}$ & - & $\mathrm{R} / \mathrm{I}$ \\
\hline 129 & Vitex altissima L.f. & Verbenaceae & - & - & - & - & 1.4754 & $\mathrm{~F}$ & - & - & - & - \\
\hline 130 & $\begin{array}{l}\text { Clerodendrum infortunatum } \\
\text { L. }\end{array}$ & Verbenaceae & 4.866 & $\mathrm{P}$ & - & $\mathrm{R} / \mathrm{I}$ & - & $\mathrm{R} / \mathrm{I}$ & 0.9491 & G & 1.935 & $\mathrm{~F}$ \\
\hline 131 & Tectona grandis $\mathrm{L}$ & Verbenaceae & - & - & - & - & 25.2553 & $\mathrm{P}$ & - & - & - & - \\
\hline 132 & $\begin{array}{l}\text { Xanthophyllum amottianum } \\
\mathrm{Wt} \text {. }\end{array}$ & Xanthophyllaceae & 15.5942 & $\mathrm{~F}$ & 25.8827 & $\mathrm{~F}$ & - & $\mathrm{R} / \mathrm{I}$ & - & - & 8.784 & $\mathrm{~F}$ \\
\hline
\end{tabular}

$\mathrm{RS}=$ Regeneration status; $\mathrm{G}=\mathrm{Good}, \mathrm{F}=\mathrm{Fair}, \mathrm{P}=\mathrm{Poor}, \mathrm{N}=$ None, $\mathrm{R} / \mathrm{I}=$ Reappearing/Immigrating

Among the species, Cinnamomum malabatrum recorded the highest density with 63.63 individuals/ha, followed by Dimocarpus longan (52.27 individuals/ha) and Nathopegia racemosa ( 50 individuals/ha). The highest basal area was shown by Canarium strictum ( $13.75 \mathrm{~m}^{2} / \mathrm{ha}$ ) followed by Dysoxylon malabaricum $\left(5.47 \mathrm{~m}^{2} / \mathrm{ha}\right)$ and Dimocarpus longan (3.37 $\mathrm{m}^{2} /$ ha). However, Canarium strictum scored the highest IVI value of 29.04, followed by Dimocarpus longan (28.21) and Dysoxylon malabaricum (25.42).

In the study area, $47.75 \%$ of species showed regeneration, $26.86 \%$ of tree species had no regenerates and $25.37 \%$ of species were recorded only in regenerating stage. Dimocarpus longan showed good regeneration (with 296.02 seedlings/100 $\mathrm{m}^{2}$ and 104.54 saplings $\left./ 100 \mathrm{~m}^{2}\right)$, whereas Dysoxylum malaabricum showed no regeneration and Canarium strictum showed poor regeneration (with 3.4 saplings $/ 100 \mathrm{~m}^{2}$ ).
Kolthodu sacred grove (SG2)

In SG2, 81 tree species belonging to 65 genera and 33 families were recorded, among which 20 (35.71\%) were singleton species and 25 were found only in regenerating stage. A density of 457.14 individuals/ha and a basal area of $67.49 \mathrm{~m}^{2} / \mathrm{ha}$ were observed in the study area. Xanthophyllum arnottianum showed the highest density (50 individuals/ha), followed by Holigarna grahamii (28.57 individuals/ha) and Dimocarpus longan (25 individuals/ha). Syzygium cumini showed the highest basal area of $15.91 \mathrm{~m}^{2} /$ ha with 12.5 individuals/ha, followed by Canarium strictum $\left(7.33 \mathrm{~m}^{2} / \mathrm{ha}\right.$ with 21.42 individuals/ha) and Xanthophyllum arnottianum (5.70 $\mathrm{m}^{2} /$ ha). Highest IVI values were scored by Syzygium cumini (28.16) followed by Xanthophyllum arnottianum (25.88) and Canarium strictum (19.87). 
Only four species (4.93\%) showed good regeneration in this grove, whereas 14 species showed poor regeneration. However, 25 tree species each (30.8\%) showed no regeneration as well as reappearing / immigrating status. Syzygium cumini (0.44 seedlings/100 $\mathrm{m}^{2}$ and 0.44 saplings $/ 100 \mathrm{~m}^{2}$ ) and Xanthophyllum arnottianum (3.125 seedlings/100 $\mathrm{m}^{2}$ and 9.82 saplings $/ 100 \mathrm{~m}^{2}$ ) showed fair regeneration, where as Canarium strictum showed poor regeneration with only 0.44 seedlings $/ 100 \mathrm{~m}^{2}$. However, Dimocarpus longan recorded highest seedling $\left(86.60 / 100 \mathrm{~m}^{2}\right)$ and sapling $\left(120.53 / 100 \mathrm{~m}^{2}\right)$ density, followed by Nathopegia racemosa (0.89 seedlings and 15.17 saplings per $100 \mathrm{~m}^{2}$ ) and Mastixia arborea (1.78 seedlings $/ 100 \mathrm{~m}^{2}$ and 31.63 saplings $/ 100 \mathrm{~m}^{2}$ ).

\section{Huduru sacred grove (SG3)}

Along with 17 singleton species and 31 species with no mature individuals, SG3 recorded a total of 74 tree species belonging to 65 genera and 34 families. Tree density and basal area of the grove were 468.18 individuals/ha and $51.73 \mathrm{~m}^{2} /$ ha respectively. Highest basal area was showed by Stereospermum colais $\left(6.85 \mathrm{~m}^{2} /\right.$ ha with 22.72 individuals), followed by Syzygium cumini $\left(6.14 \mathrm{~m}^{2} / \mathrm{ha}\right.$ with 4.54 individuals/ha) and Hydnocarpus pentandra $\left(5.27 \mathrm{~m}^{2} /\right.$ ha with 29.54 individuals/ha). However, higher tree density was observed by an entirely different set of species: highest value was observed in Mallotus tetracoccus (86.36 individuals/ha), followed by Tectona grandis (72.72 individuals/ha) and Grewia tiliifolia (38.63 individuals/ha). Higher IVI values were scored by Mallotus tetracoccus (36.75) followed by Tectona grandis (25.25) and Hydnocarpuspentandra (23.18).

Only $36.47 \%$ of the total species recorded in SG3 showed regeneration, of which $6.75 \%$ of species had good regeneration, $12.16 \%$ had fair regeneration and $17.56 \%$ of species had poor regeneration. A highest of $41.89 \%$ of tree species were recorded only at regenerating stage and $21.62 \%$ of species had no regenerates at all. Regeneration density was dominated by Dimocarpus longan in this grove, with a value of 346.02 seedlings and 157.35 saplings per $100 \mathrm{~m}^{2}$ area. Mallotus tetracoccus showed no regeneration even though it had the highest tree density in the study area; Tectona grandis showed poor regeneration and Hydnocarpus pentandra showed fair regeneration.

\section{Thaila sacred grove (SG4)}

A total of 72 tree species belonging to 61 genera and 32 families were recorded at SG4, among which 17 were singleton species and 18 were only in reappearing / immigrating stage. Basal area and tree density of the study site recorded to be $85.65 \mathrm{~m}^{2} / \mathrm{ha}$ and 453.33 individuals/ha respectively. Highest IVI values were scored by Hydnocarpus pentandra (25.51) followed by Syzygium cumini (25.25) and Cleidion javanicum (20.61). However, the highest density was showed by Cleidion javanicum (48.33 individuals/ha) and highest basal area was recorded by Syzygium cumini $\left(16.18 \mathrm{~m}^{2} / \mathrm{ha}\right)$.

No regeneration was observed in $31.08 \%$ of total species in this site, while $24.32 \%$ of species were reappearing/immigrating in the area. A total of $44.57 \%$ of species showed regeneration, of which $18.91 \%$ had poor regeneration. Hydnocarpus pentandra showed fair regeneration, Syzygium cumini showed no regeneration and Cleidion javanicum showed good regeneration in the study area. Overall, the lowest seedling and sapling density was observed in this sacred grove compared to other study areas. Highest seedling and sapling density was recorded by Reinwardtiodendron anamalainse (30.41 seedlings $/ 100 \mathrm{~m}^{2}$ and 39.16 saplings $/ 100 \mathrm{~m}^{2}$ ).

\section{Birunani sacred grove (SG5)}

The lowest species richness was observed in SG5 with 60 tree species belonging to 54 genera and 32 families, of which $47 \%$ (18 species) were singleton species and 22 species had no mature individuals. Values for basal area and tree density per ha were $73.84 \mathrm{~m}^{2} / \mathrm{ha}$ and 492.85 individuals/ha respectively. Dimocarpus longan showed the highest IVI value (51.13), basal area $\left(17.76 \mathrm{~m}^{2} / \mathrm{ha}\right)$ and density (92.85 individuals/ha). Vateria indica showed the second highest basal area $\left(10.58 \mathrm{~m}^{2} / \mathrm{ha}\right.$ with 39.28 individuals $\left./ \mathrm{ha}\right)$ and Caryota urens showed second highest density (57.14 individuals/ha with a basal area of $2.29 \mathrm{~m}^{2} / \mathrm{ha}$ ).

In SG5, $43.32 \%$ of species showed regeneration, of which $21.66 \%$ had poor regeneration. However, $20 \%$ of recorded species had no regenerates and $36.66 \%$ of species were found only in regenerating stage. Tree species with high IVI values also showed good regeneration. Among the five sacred groves, SG5 recorded higher regeneration density, with a value of 855.35 seedlings $/ 100 \mathrm{~m}^{2}$ and 641.96 saplings $/ 100 \mathrm{~m}^{2}$. Vateria indica showed a good regeneration with 466.07 seedlings $/ 100 \mathrm{~m}^{2}$ and 217.85 saplings $/ 100 \mathrm{~m}^{2}$.

Among the five study sites, higher CDI value was recorded for SG1 (42.85\%). Temple construction and the effect of pilgrims were observed in SG1. Grazing, presence of weeds and footpath were common in all the sacred groves. Basal area showed inversely proportional relationship with CDI values of the studied groves. However, species richness and densities (seedling, sapling and tree density) did not show a clear relation with CDI values per se (Fig. 5a and 5b).

\section{Discussion}

Depending on climatic, edaphic and biotic factors (Ayyappan and Parthasarathy, 1999), species richness

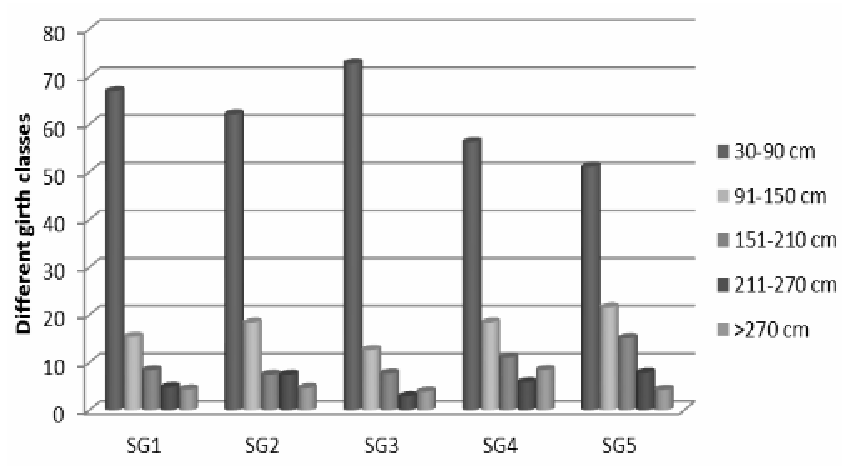

Fig. 2. Distribution of girth classes among the sacred groves, Virajpet 
366

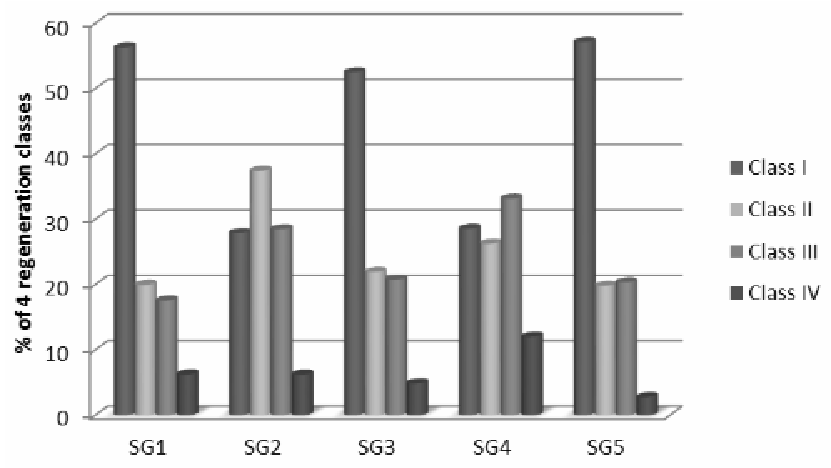

Fig. 3. Distribution of regeneration classes among the sacred groves, Virajpet

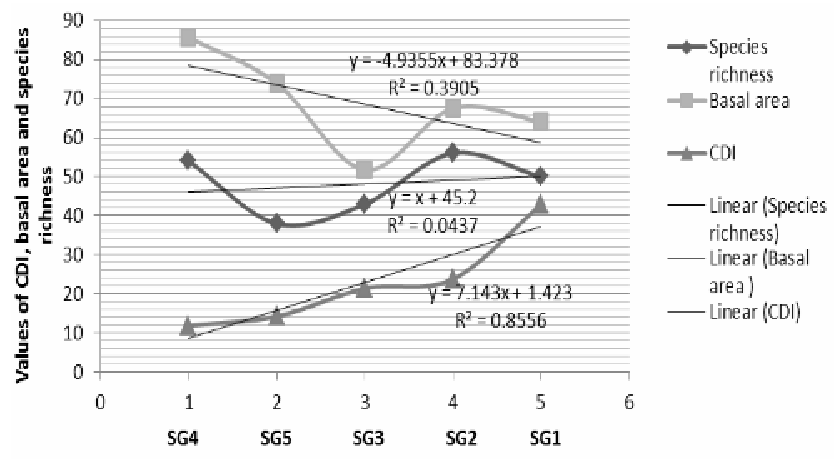

Fig. 5a. CDI values in terms of species richness and basal area in the sacred groves of Virajpet

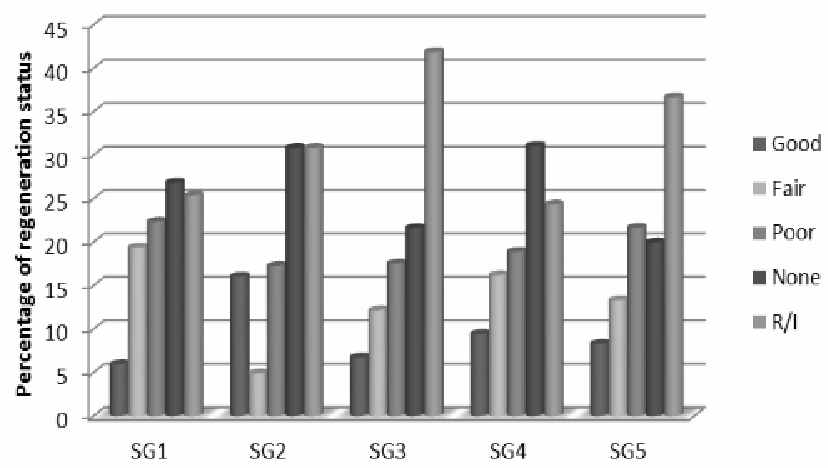

Fig. 4. Distribution of percentage of regeneration status of tree species in the five sacred groves, Virajpet

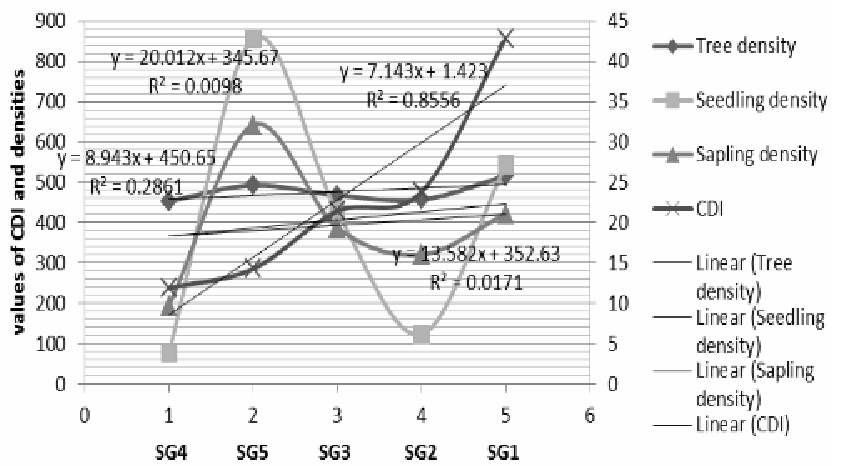

Fig. 5b. CDI values in terms of densities in the sacred groves of Virajpet

Table 3. Species richness, indices and density values of the five sacred groves

\begin{tabular}{|c|c|c|c|c|c|c|}
\hline No & Parameters & SG1 & SG2 & SG3 & SG4 & SG5 \\
\hline 1 & No. of species & $67\left(50^{a}+17^{b}\right)$ & $81\left(56^{a}+25^{b}\right)$ & $74\left(43^{a}+31^{b}\right)$ & $72\left(54^{a}+18^{b}\right)$ & $60\left(38^{a}+22^{b}\right)$ \\
\hline 2 & No. of genera & 56 & 65 & 65 & 61 & 54 \\
\hline 3 & No. of families & 33 & 33 & 34 & 32 & 32 \\
\hline 4 & Shannon's index & 3.341 & 3.577 & 3.025 & 3.633 & 3.038 \\
\hline 5 & Simpson's index & 0.9456 & 0.9613 & 0.9177 & 0.9653 & 0.9223 \\
\hline 6 & Pielou's index & 0.8542 & 0.8887 & 0.8043 & 0.9107 & 0.8353 \\
\hline 7 & Basal area $\mathrm{m}^{2} / \mathrm{ha}$ & 64.145 & 67.4955 & 51.73 & 85.65 & 73.84 \\
\hline 8 & Tree density/ha & 515.9 & 457.14 & 468.18 & 453.33 & 492.85 \\
\hline 9 & Seedling density $/ 100 \mathrm{~m}^{2}$ & 543.18 & 124.107 & 428.4 & 77.5 & 855.35 \\
\hline 10 & Sapling density $/ 100 \mathrm{~m}^{2}$ & 422.15 & 320.98 & 388.06 & 193.75 & 641.96 \\
\hline
\end{tabular}

a: the number of tree species with mature individuals in the sacred grove; $b=$ the number of tree species without mature individuals in the sacred grove.

Table 4. Sorenson's similarity index values (\%) for the five sacred

\begin{tabular}{cccccc}
\hline & SG1 & SG2 & SG3 & SG4 & SG5 \\
\hline SG1 & ${ }^{*}$ & & & & \\
SG2 & 64.86 & ${ }^{*}$ & & & \\
SG3 & 62.41 & 65.8 & ${ }^{*}$ & & \\
SG4 & 57.55 & 69.28 & 69.86 & $*$ & \\
SG5 & 59.84 & 65.24 & 67.16 & 62.12 & $*$ \\
\hline
\end{tabular}

develops in an ecosystem. It is advocated that species richness of an ecosystem results from its complexity, variability and productivity. Therefore, diversity tends to increase as the environment becomes more favourable and more predictable (Putman, 1994). From the present investigation it was evident that, apart from the geological and climatic conditions, protection over a long period of time provided by local communities on the basis of socio-cultural belief system has an instrumental role in tree species richness, their distribution and regeneration in the studied sacred groves. Similar results, with high species richness within sacred groves has been reported from various researchers across the country (Ramakrishna et al., 1998; Malhotra et al., 2001; Ramanujam and Cyril, 2003; Khan et al., 2008), upholding the significance of the role of these areas in conservation of biodiversity.

Higher similarity $(64.41 \%)$ between the sacred groves as indicated by the Sorenson's similarity index could be attributed to the similar geographical and climatic conditions. Shannon's diversity index values (3.02-3.63) for the five sacred groves were within the range reported by various workers for the Western Ghats region: Jayakumar and Nair (2013) reported a range of 2.79 to 3.67 for New Amarambal reserve forest of Nilgiri Biosphere Reserve, Kerala; Vasanthraj and Chandrashekar (2006) reported a value of 4.9 for Charmadi reserve forest, Karnataka; Kanade et al. (2008) recorded 2.58 for Chandoli National Park, North Western Ghats, Maharastra; Gunaga et al. (2013) recorded a range of 2.55 to 3.48 in the Kaans of Uttara Kannada district, 
Karnataka. Simpson's index value of 0.9424 for the present study was found to be well in agreement with a few studies existing, as reported for Western Ghats where it ranged from 0.93 to 0.73 (Bhat and Kaveriappa, 2009), while for sacred groves of Meghalaya ranged from 0.95 to 0.93 (Upadhyaya $e t$ al., 2003) and in the forests of Little Andaman Simpson's index ranged from 0.97 to 0.56 (Rasingam and Parthasarathy, 2009).

Basal area of tree species is an important indicator of tree stocking which reflects biomass and carbon stock capacity of them. Significantly higher values recorded for the present study areas indicate their importance. Gunaga et al. (2013) reported a value of $22.60-48.80 \mathrm{~m}^{2} / \mathrm{ha}$ basal area for the Kaans of Uttara Kannada district, Karnataka; Parthasarathy (2001) reported a value of $55.34-78.32 \mathrm{~m}^{2} /$ ha for KalakadMundanthurai Tiger Reserve forest, Western Ghats; Jayakumar and Nair (2013) recorded a value of $48.00 \mathrm{~m}^{2} / \mathrm{ha}$ for Evergreen forests of New Amarambalam Reserve Forests, Western Ghats; Muthuramkumar et al. (2006) recorded 46.3-99.67 $\mathrm{m}^{2} / \mathrm{ha}$ of basal area for Anamalai hills forest, Western Ghats and Tripathi $e t$ al. (2010) reported a value of $35.12 \mathrm{~m}^{2} / \mathrm{ha}$ for forests of East Khasi Hills.

Density of tree species and girth class distribution among the studied groves was found to be appreciably good. In comparison with other study reports such as a density of 999 stems/ha for sacred forests of Chittoor, Andhra Pradesh (Rao et al., 2011), 387 stems/ha in the Kaan forests of Shimoga (Gunaga et al., 2013), a range of 384-566 stems/ha of area for Valparai, Annamalai hills, Tamil Nadu (Muthukumar et al., 2006), the present investigation recorded notably higher values. A higher percentage of individuals in the lower girth in the present study sites indicate a lower disturbance, such as extraction of younger individuals for poles and firewood purpose. Social system of beliefs and taboos pertaining to these sacred groves have protected them from such anthropogenic activities.

The future tree species composition could be predicted from the relative number of present tree species seedlings and saplings. Overall, a good number of regeneration was recorded from these forest patches. A reverse J-shaped curve for different regeneration classes observed in the current studied groves means that the maximum number of regenerates counted for class I and the minimum number of regenerates for class IV. This reverse Jshaped curve is an indication of sustainable regeneration in the forest ecosystem. Prasad and Nageeb (2012) reported reverse J-shaped curves for regeneration in the eight Dipterocarp forests of Western Ghats, Karnataka. Boraiah et al. (2003) reported a similar curve for reserve forest, conserved and disturbed sacred groves of Kodagu. A population structure characterized by the presence of a sufficient number of seedlings, saplings and young trees implies satisfactory regeneration behaviour, while an inadequate number of seedlings and saplings of tree species in a forest indicates poor regeneration (Nazir et al., 2013).

In the present study highest percentage of species showed regeneration (42.07\%) followed by reappearing/immigrating (31.82\%) status. On the other hand, the number of tree species $(26.08 \%)$ with no regeneration was recorded to be high. This could be due to a more or less consistent dominance of Dimocarpus longan in tree density, seedling density and sapling density values observed within all five sacred groves, which might be a cause of concern. Higher percentage of species found within the groves as reappearing or immigrating from other areas could be correlated to the fact that all the five study areas are surrounded by coffee plantations and agricultural fields. Hence, they serve as roosting/resting habitats for birds and animals, which cause the dispersal of seeds from other areas. In the sacred grove of Manipur (Khumbongmayum et al., 2006) a greater number of tree species had good and fair regeneration, followed by reappearing species and a very few species showed no regeneration. The authors opined that the presence of species that are represented only by adults in these sacred groves of Manipur could be due to their poor seed set, germination and establishment of seedlings in the forest. Nazir et al. (2013) reported that overall maximum percentage of species showed no regeneration followed by fair, good and poor regeneration for sacred and protected landscapes of Garhwal Himalaya, India, and opined that this could be a cause of concern in the future.

Evaluation of regeneration status of each species in all the sacred groves revealed that the density of mature individuals and regenerates were not proportionate. Chauhan et al. (2008) opined that the regeneration of a species does not account for its adult density, meaning there is no linear relationship between seedling density and adult density of a species. Jones et al. (1994) opined that seedling layer in various forests differs in composition from their respective overstories. Similar results were observed for the present study areas where the density of mature individuals did not have proportional effect on density of regenerates. For example, in the case of SG3 - Mallotus tetracoccus, with the highest density, showed no regeneration and Tectona grandis with the second highest tree density showed poor regeneration, while Dimocarpus longan with just 13.63 individuals/ha had 346.02 seedlings and 157.95 saplings/100 m². In SG5, Caryota urens had the second highest density, but its regeneration status was found to be poor, without any seedlings. Khumbongmayum et al. (2006) opined that species diversity and population structure will be stable or reduced and regeneration potential will be negligible if the number of species represented only by adults becomes higher in any forest.

Survival rates of seedlings and saplings depend upon canopy openness (Nagamatsu et al., 2002), microenvironmental conditions, natural light gaps (Welden $e t$ al., 1991), soil, temperature and topography. In the present study, lower seedlings and sapling density observed in SG4 as compared with other groves could be attributed to lower light penetration as a result of closed canopy in the study area. Seedling and sapling density of tree species in the studied groves ranged from 77.5-855.85 seedlings $/ 100 \mathrm{~m}^{2}$ and 193.75-641.96 saplings $/ 100 \mathrm{~m}^{2}$. Relatively, these values are higher than other study results such as: Pokhriyal et al. (2010) reported a seedling density of 3.70 to $7.01 / 100 \mathrm{~m}^{2}$ and a sapling density of 23.29 to $34.96 / 100 \mathrm{~m}^{2}$ in the watersheds of Garhwal Himalaya. Khumbongmayum et al. (2006) reported a range of 3415-8803 seedlings/ha and 1943-4540 saplings/ha in the sacred groves of Manipur. Chauhan et al. (2010) recorded 158.7 seedlings and 496.0 saplings per hectare respectively in Terai-Bhabhar of Sohagibarwa Wildlife Sanctuary, India. Nazir et al. (2013) reported a 
368

seedling density ranging from 11.36 to 18.74 seedlings/100 $\mathrm{m}^{2}$ and sapling density from 8.84 to 15.2 saplings $/ 100 \mathrm{~m}^{2}$ in the sacred groves of Garhwal Himalayas.

\section{Conclusions}

Even though sacred groves are protected on the basis of local beliefs and taboos, they are not immune from human activities. For example, sanskritization or replacement of nature worship system into idol worship, construction of concrete temples and celebration of festivals is becoming a common trend in many of the sacred groves, attracting large number of pilgrims. This is leading to unsustainable development, involving clearance of forests for road construction and indiscriminate dumping of wastes in natural groves. During the current investigation, it was observed that sanskritization, mud roads, footpath and grazing were found to be common disturbing factors in these sacred groves. The mild disturbance has favoured the overall species richness. A good regeneration of tree species among these sacred groves was witnessed with a significant difference in regenerating and mature tree species composition. As these sacred groves abode a rich diversity of arboreal species with good regeneration potential, prime importance should be given for their conservation.

\section{Acknowledgements}

Authors are thankful to the RFOs, Virajpet, Ponnampet and Thithimathi, and their staff for help and support during the field work. The author (Shwetha) is thankful to DST, Ministry of Science and Technology, Government of India for INSPIRE Fellowship.

\section{References}

Ayyappan A, Parthasarathy N (1999). Biodiversity inventory of trees in a large scale permanent plot of tropical evergreen forest at Varagalaiar, Anamalais, Western Ghats, India. Biodiversity and Conservation 8:1533-1554.

Bhat PR, Kaveriappa KM (2009). Ecological studies on Myristica swamp forests of Uttara Kannada, Karnataka, India. Tropical Ecology 50(2):329-337.

Boraiah KT, Vasudeva R, Shonil AB, Kushalapa CG (2003). Do informally managed sacred groves have higher richness and regeneration of medicinal plants than state managed reserve forests? Current Science 84:804-808.

Chauhan DS, Bhupendra Singh, Shashi Chauhan, Dhanai CS, Todaria NP (2010). Regeneration and plant diversity of natural and planted sal (Shorea robusta Gaertn.F.) forests in the TeraiBhabhar of Sohagibarwa Wildlife Sanctuary, India. Journal of American Science 6(3):32-45.

Chauhan DS, Dhanai CS, Bhupendra Singh, Shashi Chauhan, Todaria NP, Khalid MA (2008). Regeneration and tree diversity in natural and planted forests in a Terai-Bhabhar forest in Katerniaghat Wildlife Sanctuary, India Tropical Ecology 49(1):53-67.

Curtis JT, McIntosh RP (1950). The interrelations of certain analytic and synthetic phytosociological characters. Ecology 31:434-455.
Devi LS, Yadava PS (2006). Floristic diversity assessment and vegetation analysis of tropical semievergreen forest of Manipur, North East India. Tropical Ecology 47(1):89-98.

Gunaga S, Rajeshwari N, Vasudeva R (2013). Tree diversity and disturbance of Kaan forests: Relics of a community protected climax vegetation in the Central Western Ghats. Tropical Ecology 54(1):117-131.

Jayakumar R, Nair KKN (2013). Species diversity and tree regeneration patterns in tropical forests of the Western Ghats, India. ISRN Ecology ID890862.

Jones RH, Sharitz RR, Dixon PM, Segal DS, Schneider RL (1994). Woody plant regeneration in four floodplain forests. Ecological Monographs 64:345-367.

Kanade R, Tadwalker M, Kushalappa C, Patwardhan A (2008). Vegetation composition and woody species diversity at Chandoli National park, northern Western Ghats, India. Current Science 95(5):637-646.

Keshavamurthy KR, Yoganarasimhan SN (1990). Flora of Coorg (Kodagu), Karnataka, India. Vismat Publishers, Bangalore.

Khan ML, Ashalata Devi K, Tripathi RS (2008). The sacred groves and their significance in conserving biodiversity - An overview. International Journal of Ecology and Environmental Sciences 34(3):277-291.

Khumbongmayum AD, Khan ML, Tripathi RS (2006). Biodiversity conservation in sacred groves of Manipur, Northeast India: population structure and regeneration status of woody species. Biodiversity and Conservation 15:2439-2456.

Malhotra KC, Gokhale Y, Chatterjee S, Srivastava S (2001). Cultural and ecological dimensions of sacred groves in India. Indian National Science Academy, New Delhi, and Indira Gandhi Rashtriya Manav Sangrahalaya, Bhopal.

Muthuramkumar S, Ayyappan N, Parthasarathy N (2006). Plant community structure in tropical rain forest fragments of the Western Ghats, India. Biotropica 38(2):143-160.

Nagamastu D, Seiwa K, Sakai A (2002). Seedling establishment of deciduous trees in various topographic positions. Journal of Vegetation Science 13:35-44.

Nazir AP, Negi AK, Yogesh Gokhale, Todaria NP (2013). Tree regeneration status of sacred and protected landscapes in Garhwal Himalaya, India. Journal of Sustainable Forestry 32(3):230-246.

Parthasarathy N (2001). Changes in forest composition and structure in three sited of tropical evergreen forest around Segaltheri, Western Ghats. Current Science 80(3):389-393.

Pascal JP, Ramesh BR (1987). A field key to the trees and lianas of the evergreen forests of the Western Ghats (India), 2nd ed. Institute of Français De Pondichéry.

Pielou EC (1969). An introduction to mathematical ecology. Wiley, New York.

Pokhriyal P, Pooja Uniyal, Chauhan DS, Todaria NP (2010). Regeneration status of tree species in forest of Phakot and Pathri Rao watersheds in Garhwal Himalaya. Current Science 98(2):171-175.

Poornika RBJ, Sathish BN, Mohana, GS, Somanna Chittiappa, Kushalappa CG (2011). Field guide - Trees of coffee agroforestry systems in Kodagu. CAFNET Project. 
Prasad AGD, Nageeb AA (2012). Floristic diversity of regenerated tree species in Dipterocarp forests in Western Ghats of Karnataka, India. Journal of Environmental Biology 33:791-797.

Putman RJ (1994). Community ecology. Chapman \& Hall, London.

Raghavendra S, Kushalappa CG (2011). Devarakadu (Sacred groves) of Kodagu: A living tradition of community linked conservation. Published by Karnataka Forest Department.

Ramakrishna PS, Saxena KG, Chandrashekara UM (1998). Conserving the sacred for biodiversity management. Oxford and Ibh Publishing Co Pvt Ltd.

Ramanujam MP, Cyril KPK (2003). Woody species of four sacred groves in the Pondicherry region of South India. Biodiversity and Conservation 12:289-299.

Rao BRP, Suresh Babu MV, Sridhar Reddy M, Madhusudhana Reddy A, Srinivasa Rao V, Sunitha S, Ganeshaiaih KN (2011). Sacred groves in Southern Eastern Ghats, India: Are they better managed than forest reserves? Tropical Ecology 52(1):79-90.

Rasingam L, Parthasarathy N (2009). Tree species diversity and population structure across major forest formations and disturbance categories in Little Andaman Island, India. Tropical Ecology 50(1):89-102.

Saldanha C J (1984). Flora of Karnataka - Vol 1. Oxford and IBH Publishing Co, New Delhi.

Saldanha C J (1996). Flora of Karnataka - Vol 2. Oxford and IBH Publishing Co, New Delhi.
Shannon CI, Weiner W (1963). The mathematical theory of communication. University of Illinois Press, Urbana, 111, USA.

Shankar U (2001). A case of high tree diversity in a sal (Shorea robusta)-dominated lowland forest of Eastern Himalaya: Floristic composition, regeneration and conservation. Current Science 81(7):776-786.

Simpson EH (1949). Measurement of diversity. Nature 163:688.

Sorensen T (1948). A method of establishing groups of equal amplitude in plant sociology based on similarity of species content. Kongelige Danske Videnskabernes Selskab 5(1-34):4-7.

Tripathi OP, Upadhyaya K, Tripathi RS, Pandey HN (2010). Diversity, dominance and population structure of tree species along fragment size gradient of a subtropical humid forest of Northeast India. Research Journal of Environmental and Earth Sciences 2(2):97-105.

Upadhyaya K, Pandey HN, Law PS, Tripathy RS (2003). Tree diversity in sacred groves of the Jaintia hills in Meghalaya, Northeast India. Biodiversity and Conservation 12:583-597.

Vasanthraj BK, Chandrashekar KR (2006). Analysis of the structure of Charmady reserve forest. Tropical Ecology 47(2):279-290.

Welden CW, Hewett SW, Hubbell SP, Foster RB (1991). Sapling survival, growth and recruitment: Relationship to canopy height in a Neotropical forest. Ecology 72:35-50. 